\title{
Le jardin " anglais » : représentation, rhétorique et translation de la nation britannique, 1688-1820
}

Representation, Rhetoric and Translation of The 'English' Garden in Britain, 1688-1820

Laurent Châtel

\section{OpenEdition}

\section{Journals}

Édition électronique

URL : http://journals.openedition.org/rfcb/1883

DOI : $10.4000 /$ rfcb.1883

ISSN : 2429-4373

Éditeur

CRECIB - Centre de recherche et d'études en civilisation britannique

Édition imprimée

Date de publication : 1 janvier 2006

ISSN : 0248-9015

\section{Référence électronique}

Laurent Châtel, « Le jardin « anglais » : représentation, rhétorique et translation de la nation

britannique, 1688-1820», Revue Française de Civilisation Britannique [En ligne], XIII-4 | 2006, mis en ligne le 25 avril 2018, consulté le 10 décembre 2020. URL : http://journals.openedition.org/rfcb/1883 ; DOI : https://doi.org/10.4000/rfcb.1883

Ce document a été généré automatiquement le 10 décembre 2020.

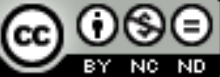

Revue française de civilisation britannique est mis à disposition selon les termes de la licence Creative Commons Attribution - Pas d'Utilisation Commerciale - Pas de Modification 4.0 International. 


\title{
Le jardin « anglais » : représentation, rhétorique et translation de la nation britannique, $1688-1820$
}

\author{
Representation, Rhetoric and Translation of The 'English' Garden in Britain, \\ 1688-1820
}

Laurent Châtel

1 Le jardin ne saurait être perçu hors de l'Histoire. Il n'en a pourtant pas toujours été ainsi. 'Those who give a great deal of loving care to the tending of trees and flowers may object to the presence of historians in their favourite haunts'. C'est à Michel Baridon que l'on doit d'avoir introduit l'histoire, l'historique et l'historicité dans les jardins, notamment dans deux articles séminaux, 'History, Myth and the English Garden' (article donné oralement puis publié à la National Gallery of Art de Washington en 1989) et 'Ruins as Mental Construct'. En convoquant de concert les notions de jardin et de nation, on choisit précisément d'inscrire la création paysagère dans une histoire, c'est-à-dire dans son contexte social, culturel et politique, mais on introduit aussi une réflexion d'ordre linguistique. En quoi ce qu'on nomme jardin anglais était-il au XVIII ${ }^{e}$ siècle l'apanage d'une nation? Déjà John Dixon Hunt en 1986 et Michel Baridon en 2000 posaient les questions respectives, 'How English was the English Landscape Garden?' et « Existe-t-il un style anglais? »'. Oserait-on aujourd'hui suggérer que le jardin anglais n'est anglais que de nom?

2 L'expression lexicalisée "jardin anglois » ou "jardin à l'anglaise » laisse croire à un rapport de contiguïté entre jardin et nation et à un reflet de l'un dans l'autre. Certes, les jardins sont présentés comme une des plus singulières contributions de la GrandeBretagne aux arts internationaux. Mais le jardin étant un prisme sur lequel convergent plusieurs regards, tout en étant dérivé d'une longue tradition à l'échelle planétaire, il paraît douteux que le jardin puisse être réduit à une nation. Le mérite d'une réflexion sur 
les modalités nationales est de démasquer le terme "jardin anglais ». En fidèle historiciste, on ne saurait sous-estimer l'importance de la réception dans la cristallisation d'une forme artistique : le spectateur, qu'il soit favorable ou critique, forge et fixe des notions qui auraient pu demeurer fluctuantes. Puisque, par rejet ou par goût, ce sont aussi des Français qui ont nommé le jardin anglais, on peut soutenir que le jardin dit « anglais» fut le reflet de la nation française autant que de la nation britannique. La transparence entre jardin anglais et nation britannique s'évanouit donc pour céder la place à un réseau complexe d'influences et de translations impliquant appropriation, discours et propagande. La brève présentation des enjeux nationaux du jardin s'attache à mettre en valeur le jardin comme représentation nationale, puis comme revendication nationaliste et enfin comme traduction.

\section{'All Nature was a garden': le jardin comme icône nationale}

Entre 1688 et 1820, la Grande-Bretagne s'est representé que sa nation tout entière était un jardin. Bien qu'il soit de rigueur en cette ère du soupçon des discours d'y déceler une rhétorique patriotique, il importe dans un premier temps d'évaluer et de comprendre un tel phénomène culturel d'identification. La modernité affichée par la Grande-Bretagne dans la seconde moitié du XVIII ${ }^{e}$ siècle fut le résultat d'une convergence entre un processus de construction identitaire lié à l'Act of Union de 1707 (trois pays se retrouvaient réunis en un seul) et la découverte des terres nationales. En effet, ces éléments «neufs »une nation récemment redélimitée et une nature nouvellement appréciée - provoquèrent une soif de progrès et de créativité. L'impératif impérial expansionniste, qui resta à l'ordre du jour, se doubla d'un agenda national de découverte géographique, géologique et esthétique des terres $\mathrm{du}$ " nouveau » royaume.

4 Véritable terra incognita, la Grande-Bretagne fit l'objet d'un proto-tourisme : au Grand Tour s'ajouta un 'Home Tour', comme si les Britanniques étaient soucieux (parfois forcés en temps de guerre) de se retrancher sur leurs propres terres. Les régions découvertes étaient les Alpes britanniques, soit le Lake District, le Yorkshire, le Derbyshire, mais aussi le Pays de Galles et l'Écosse. Or, ce mouvement de découverte des 'beauties' de la GrandeBretagne s'accompagnait parallèlement d'un goût pour le jardin. Les textes de Joseph Addison sur la nécessité d'un double regard sur la terre, tant esthétique qu'économique, permirent sans doute d'unir dans un même ensemble jardins, terre agraire et merveilles de la nature. Horace Walpole tenta de communiquer l'enthousiasme provoqué par cette prise de conscience:

Enough has been done to establish such a school of landscape, as cannot be found on the rest of the globe. If we have the seeds of a Claude or Gaspar amongst us, he must come forth. If wood, water, groves, valleys, glades, can inspire or poet or painter, this is the country, this is the age to produce them. The flocks, the herds, that now are admitted into, now graze on the borders of, our cultivated plains, are ready before the painter's eyes, and group themselves to animate his picture. ${ }^{2}$

Le génie de la nation se confond ici avec le génie du lieu. Nature, campagne, terres, land et landscape se confondent dans le jardin qui devient substantifique moëlle, essence de la nation. Les théories philosophiques de l'humanisme civique confirmèrent l'aristocratie dans la haute idée qu'elle se faisait de ses terres ${ }^{3}$ et les spécialistes des élites foncières et du patrimoine rural ont bien montré l'importance de la conscience de propriétaire ${ }^{4}$. La 
nation fut conçue en termes spatiaux, opposant vues lointaines et panoramiques (donc perspective élevée) aux vues rapprochées et confinées (donc limitées) ${ }^{5}$.

Une telle identification de la Grande-Bretagne avec le jardin est le signe d'une conscience paysagère éminente, ou pour parler comme Augustin Berque d'une "civilisation paysagère » mûre ${ }^{6}$. Le jardin servit la définition d'une nation unie autour du respect et du goût pour la nature. De même qu'on date le concept de patrimoine et de protection légale des sites et des monuments du XIX siècle, grâce à des utopistes tels que Ruskin et Morris, de même pourrait-on soutenir que la culture patrimoniale verte, agricole comme esthétique, prit racine au XVIII siècle - un culte et une culture de la terre incarnés par la personne même du roi, 'Farmer George'. Bien avant l'idée de cités-jardins, c'est l'idéal d'une «nation-jardin » qui constitua l'image de marque de la Grande-Bretagne. Face à «L'État, c'est moi », les Britanniques proclamaient «Le jardin, c'est nous ». D'aucuns s'étonnent de la survivance d'une telle image, qui est l'apanage de l'élite: 'Like many commentators, before and since, Repton was convinced of the decline and imminent fall of landed culture. Writing two centuries later I am struck by its resilience.? Mais comment un cultural historian peut-il nier, même s'il la déplore, la réalité culturelle d'un mythe national fondateur et fédérateur ? L'exemple de la monarchie britannique est probant : contestée, critiquée, déclarée moribonde, elle demeure néanmoins un élément constitutif de la fibre nationale. Que J.M.W. Turner, avec son Fighting Temeraire, ait remporté le référendum organisé par la BBC en juillet-août 2005, Vote for Britain's Favourite Painting, indique que cette mentalité paysagère demeure ancrée dans la psyché nationale. L'importance culturelle du gazon, de la verdure, des pépinières et du National Trust auprès des classes moyennes aux $\mathrm{XX}^{\mathrm{e}}$ et $\mathrm{XXI}^{\mathrm{e}}$ siècles est profonde en Grande-Bretagne, et, même si l'effervescence liée au jardin n'est pas exclusive à ce pays, on ne saurait la comparer à la furor hortensis française (publications aussi bien que jardinage), phénomène récent. La coïncidence « jardin anglais » est donc indéniable puisqu'elle est le fruit d'une volonté de cristalliser un savoir-faire, une distinction et une différence.

\section{Jardin « anglais » et anglicité : une image partielle de la nation?}

7 Si l'on se devait de reconnaître une curiosité, un authentique talent et une intense créativité de la nation britannique, on se doit aussi de savoir si le jardin anglais fut un reflet entier de la nation. Or, ce qui est gommé dans le concept de jardin anglais, c'est tout d'abord l'idée plurielle de nation(s). Le jardin anglais est une étiquette donnant à voir une unité qui est trompeuse. Comme l'écrit Linda Colley:

[...] the process whereby three different countries - England, Wales, and Scotland acquired some common sense of Britishness between 1707 and 1837 was not a gradual, or an even, or a pre-determined one. Britishness was a surprisingly rapid and always partial invention, which [...] never obliterated other, older attachments.

8

8 L'anglicité du jardin est mise à l'épreuve dès lors que l'on tient compte des particularismes nationaux gallois et écossais. 'Inside Great Britain lurked little England'. ${ }^{9}$ Et si le jardin anglais était "anglais» précisément parce qu'il ne fut pas britannique? L'Angleterre forgea une image qu'elle souhaita emblématique des trois nations, mais qui, ironiquement, ne fit pas honneur au génie du lieu et dont la formule générique dissimula les particularités de la créativité déployée à l'échelle locale. La thèse de A.A. Tait est que 
le jardin paysager demeura un idiome étranger en Écosse au XVIII siècle, sans résonance culturelle ou politique comme en Angleterre, et ne fut adopté que par respect de la mode, vite remplacé par un style dit régulier qui renouait avec une tradition antérieure ${ }^{10}$.

En outre, le jardin « anglais » ne fut pas nécessairement lié à la nationalité britannique et ne fut pas toujours une créativité au nom de la nation. On en a pour preuve le cas de Guillaume III et Marie : appelés par le Parlement, ils vinrent servir la nation en 1688 mais furent les mécènes d'une créativité anglaise qui empruntait des savoir-faire hollandais. $\mathrm{Si}$ le monarque se tourna - sans aucun doute pour faire bonne mesure - vers un pépiniériste anglais (George London) et des architectes anglais (William Talman, Hawksmoor), les jardiniers demeuraient hollandais : Hendrick Quellingburgh, Samuel van Staden, Casper Gamperle et Hendrick Floris, tandis que le surintendant général des jardins royaux était Hans Willem Bentick, naturalisé Earl of Portland ${ }^{11}$. Certes, David Jacques et Arend Jan van der Horst ont montré que le goût hollandais était un mythe et qu'il est bien difficile de définir un style véritablement hollandais en Angleterre sous le règne de Guillaume III $^{12}$. Par ailleurs, les courtisans de Guillaume III affectionnaient particulièrement les dessins de Perrault, Marot et Lenôtre et un des architectes qui redessinèrent le grand parterre d'Hampton Court fut Daniel Marot, un huguenot qui avait émigré à La Haye ${ }^{13}$. Ce qui caractérise le jardin anglais prôné par la nation après 1688, c'est donc moins une promotion du génie local « anglais » que le cosmopolitisme :

An eloquent testament to the craving to emulate French achievements, was the stream of French designers - de Caus, Mollet, Beaumont, Marot, Tijou and even Le Nôtre - who worked in or advised upon English gardens [...]. Without denying that distinctive national characteristics existed, which were more marked amongst the second-rank houses, gardens at courtier level in Britain and the Netherlands drew inspiration from the same Franco-Italianate models. ${ }^{14}$

La Révolution glorieuse, qui apporta le rétablissement de la confiance politique et un élan économique, ne s'accompagna pas d'un mécénat artistique d'idiomes strictement britanniques. Wesbury Court (1696-1705), dans le Gloucestershire (National Trust), fut bien un jardin anglais moderne, mais stylistiquement, par ses formes qui font écho à des pratiques hollandaises, son image renvoyait moins le promeneur à des caractéristiques nationales qu'à la Hollande. On peut donc dire que tant que le jardin afficha une référentialité étrangère (qu'elle fût italienne, française ou hollandaise), il ne servait pas la nation britannique, bien qu'il fût une illustration de la créativité du pays. Il n'y eut donc pas adéquation entre la défense des intérêts nationaux (ce pourquoi Marie et son époux furent « invités ») et l'image horticole donnée à la nation. Le jardin «anglais » n'est pas né de la Glorious Revolution et d'un respect des libertés, puisque le culte de la liberté emblématisé par la signature du Bill of Rights n'engendra pas une esthétique horticole " affranchie ». Il fallut une prise de conscience culturelle et patriotique pour que se mette en place, ex nihilo, un "projet» de formule qu'on appellerait "anglaise» et qui, a posteriori, semblerait neuve. Ainsi la détermination de voir naître un jardin national naquit avec le règne de la reine Anne (1702-1714), qui afficha publiquement un rejet des pratiques de son beau-frère, Guillaume III, et promut un style « libre » à l'image de sa nation, véritable réaction contre la manière hollandaise ('the Dutch Taste, which came in with the revolution ${ }^{15}$ ). Le jardin anglais, moins émanation de la nation, qu'élaboration de toutes pièces d'une formule éclectique à l'anglaise.

11 Par ailleurs, il est raisonnable de penser que le jardin anglais ne fut pas la préoccupation de toute la population. Ce fut une représentation nationale sur fond de nonreprésentation parlementaire. La représentation par voie du jardin supplanta-t-elle pour 
un temps la représentation par la voix du suffrage ? On sait que les grands domaines ont toujours été ouverts à des heures précises pour la visite ; la vente de guides de jardin l'atteste. En outre, les théories esthétiques et psychologiques dominantes avaient rendu possible l'appropriation personnelle d'une propriété étrangère par l'intermédiaire des cinq sens. Le promeneur pouvait jouir du jardin librement sans souffrir de lacunes intellectuelles puisque l'appel à la raison céda progressivement la place à une mobilisation sensualiste ${ }^{16}$. Toutefois, l'idée que le jardin fut la chose la mieux partagée en Angleterre - véritable icône démocratique - est relative puisqu'il n'est pas prouvé dans les faits que le commun des mortels entrait dans les jardins et en jouissait effectivement. Tout au plus pourrait-on s'appuyer sur l'ermite employé par la reine Caroline pour son ermitage dans les années 1730, Stephen Duck, ancien agriculteur devenu poète, "by art unaided. ${ }^{17}$ Certes, les témoignages sur la fréquentation des jardins publics révèlent une mixité et une coexistence inattendue de classes sociales, notamment à Londres dans les parcs de St James, de Vauxhall et de Ranelagh. Il manque à ce jour dans les études sur la réception un volet sociologique : il resterait à prouver que les jardins privés, comme ce fut le cas plus tard pour les musées, contribuèrent à la cohésion sociale de la nation. Les classes laborieuses, qui furent littéralement des zones d'ombre dans le paysage - 'the dark side of the landscape $e^{18}$ - ne semblent pas avoir eu « droit de jardin $»^{19}$ :

This ideology opened up control over nature to all men, regardless of blood, lineage and nobility, while restricting it to those who possessed the property, education and refinement to make the correct 'associations' and the land upon which to reveal their taste. ${ }^{20}$

Mais après 1789, et surtout après la Terreur, l'apparente unité de la nation autour d'une représentation paysagère commune fut brisée; la fin du règne de Georges III est marquée par une remise en cause de la théorie de l'improvement : la valeur positive, utilitaire du jardin paysager fut soumise à de sévères critiques qui dénoncèrent ces dépenses luxueuses faites à titre privé et non public. Le premier architecte paysager professionnel, Repton, ressentit une tension grandissante entre conscience sociale, budgets modestes et création de jardin dans l'exercice même de sa fonction ${ }^{21}$. En 1816, William Cobbett se fit quant à lui l'écho de l'inadéquation de l'image nationale :

[I recall] a book that we used to look at a great deal entitled 'A Picture of England'. It contained views of Country Seats and of fine hills and valleys...Alas! This was not a picture of England, if by England, we mean anything more than a certain portion of houses, Trees and Herbage. If, by England, we mean the English nation; and if, by the nation we mean the great body of the people..$^{22}$

S'il y eut un vif engouement pour le jardin en Angleterre, il nous faudrait connaitre les formes des jardins populaires pour estimer objectivement l'envergure nationale de la pratique paysagère.

\section{'A Patriotick Progress?': le jardin comme représentation « nationaliste »}

13 Le jardin anglais revêtit une dimension véritablement nationale lorsqu'il fut l'objet d'une mise en scène, sinon «nationaliste ${ }^{23}$, du moins patriotique. Deux mécénats distincts, mais intimement liés, peuvent être identifiés en la personne du monarque et parmi les membres de l'élite gouvernante. L'idée toute faite d'une totale absence de mécénat étatique en Grande-Bretagne a parfois en effet occulté le réel soutien aux arts. Or sous Georges II et Georges III, le jardin fit l'objet d'un mécénat national, c'est-à-dire qu'il fut 
soutenu par la famille royale au nom de la nation, comme le furent l'architecture, la peinture et la sculpture. Georges III orchestra consciemment un patriotisme artistique et se servit des arts, de l'agriculture et de l'industrie pour promouvoir le progrès de la nation. Holger Hoock soutient la thèse $\mathrm{e}^{24}$ d'un effort concerté de la monarchie et du Parlement pour financer et défendre la cause des artistes britanniques L'étude systématique du mécénat "paysager» et de l'instrumentalisation du jardin par la monarchie et le pouvoir en place reste à faire ${ }^{25}$. On sait que les monarques ont toujours eu un Royal Gardener, mais en quoi se servit-on consciemment des domaines royaux au XVIII siècle pour promouvoir un genre national ? Dans les années 1730, l'épouse de Georges II, Caroline, ainsi que son fils Frederick, œuvrèrent à la modernistation du style des jardins de la couronne (Hampton Court, Richmond, Kew Gardens et Carlton House). Le 23 décembre 1734, un des plus célèbres témoignages de l'activité paysagère de Frederick, prince de Galles, l'associe à une diffusion nationale d'un nouveau type de jardin :

There is a new taste in gardening just arisen, which has been practised with so much success at the Prince's garden in Town, that a general alteration of some of the most remarkable gardens in the kingdom is begun, after Mr Kent's notion of gardening, viz. to lay them out, and work without either level or line. [...] The celebrated gardens of Claremont, Chiswick and Stowe are now full of labourers, to modernise the expensive works finished in them, ever since every one's memory. ${ }^{26}$

14 À Carlton House, avec l'aide de William Kent, et sous l'influence du jardin de Pope à Twickenham, le prince de Galles réussit en effet à imprimer un style qu'il tentera d'appliquer à Kew ensuite entre 1749 et 1751, date de son décès. Ce sont ensuite respectivement son épouse, Augusta, et sa mère, Caroline, qui continuèrent à soutenir le goût moderne. L'incidence pionnière du prince de Galles sur les jardins en GrandeBretagne a été relevée par les contemporains à son décès, comme l'ont noté John Harris et Michael Snodin :

Gardening and planting have lost their best friend and encourager; for the prince had delighted in that rational amusement a long while; but lately he had had the laudable and princely ambition to excel all others.

Planting and Botany in England would be the poorer for his passing. ${ }^{27}$

À Richmond, la dimension patriotique est encore plus frappante et fut étudiée par Judith Colton : la reine Caroline mit en œuvre un programme iconographique qui emblématisait la nation par le choix d'un style, d'une mythologie et de figures nationales. Deux fabriques, aujourd'hui disparues, furent commandées à Kent, et choisies dans un style primitif, rugueux et "gothique ": un ermitage et une grotte (surnommée la grotte de Merlin). Les matériaux utilisés et les références convoquées associaient la reine à une tradition de liberté qui fit sa renommée et déclencha un discours patriotique ${ }^{28}$ :

When her Majesty consecrated these dead Heroes, she built herself a Temple in the Hearts of the People of England; who will, by this instance of her Love to Liberty and publick Virtue, think their Interests as safe in the Hands of the Government as their own. ${ }^{29}$

[...] o Britain blest to see

Thy Queen declare for Truth and Liberty. ${ }^{30}$

Hommage aux hommes de "sens ", avec cinq bustes de Rysbrack - Boyle, Locke, Newton, Samuel Clarke et William Wollaston -, l'ermitage de la reine Caroline rassemblait des éléments de fierté nationale liée à la science et à la théologie ; sans être ouvertement une propagande politique, la grotte réussit à fondre trois éléments en un : le génie de la nation, la retraite dans le jardin et l'acte de contemplation. Le journal Free Briton, le 16 août 1733, complimenta la reine, qui avait su choisir des figures nationales, en dépit de 
son grand attachement à Leibniz ${ }^{31}$. À Kew Gardens, le prince de Galles, puis la princesse Augusta, sous la direction de Lord Bute, projetèrent une transformation qui comprenait en 1753 une maison chinoise, un lac, de grandes serres et une wilderness de 50000 plantes. Mais c'est lorsque William Chambers fut nommé architecte du futur roi Georges III en 1757 que Kew devint l'instrument d'une propagande nationale et impériale. En six ans, et au prix d'un investissement somptuaire, de nombreuses fabriques "relevèrent " la platitude du terrain de Kew : un temple de Pan et d'Aréthuse, l'Alhambra, le temple de la Victoire, un temple du Soleil inspiré des Ruins of Baalbec (1757) de Robert Wood, une pagode chinoise, l'arche en ruine, la cathédrale " gothique » de Muntz, des serres et un théâtre. En (s') investissant autant, le monarque prouvait la supériorité de la science horticole et jardinière de la Grande-Bretagne, comme le note Richard Drayton : 'A king, $a$ statesman, or a colonial governor could also come to be construed as a divinely appointed gardener. ${ }^{32}$

17 Parallèlement aux jardins et parcs royaux, certains jardins privés furent des instruments sociaux, culturels et politiques. La force de la nation reposait sur la puissance d'évocation de certains jardins, fleurons des possédants (Castle Howard, Houghton Hall, Harewood, Stowe, Studley Royal). Ainsi la création de Studley Royal peut être interprétée comme un geste patriotique : le propriétaire William Aislabie dut fuir Londres et le gouvernement à la suite de la banqueroute de la South Sea Bubble pour mieux se retirer dans l'otium. Le jardin comme retraite est une première forme de "commentaire » sur l'éventuel risque de décadence d'une nation et memento mori de l'impératif de progrès. Le jardin servit l'élite gouvernante et devint ce que John Summerson a appelé 'the flaunting symbol of territorial whiggery'. C'est Stowe, en tant que jardin le plus visité d'Angleterre entre 1751 et 1820, qui servit de vitrine à la nation. Le propriétaire Richard Temple, Lord Cobham, surnommé par Jonathan Swift 'the greatest Whig in the Army', se trouvait au cœur de la nation par ses affiliations sociales et politiques, tout en étant sur les marges puisque son poste dans l'armée lui fut retiré à deux reprises, en 1714 par le gouvernement tory et en 1733 par le gouvernement whig. C'est donc dans la texture même de son domaine qu'il afficha son allégeance à la nation, tantôt en rappelant la gloire du passé national, tantôt en critiquant les écarts de la nation contemporaine. On peut dire qu'il mit en acte l'injonction de Pope en 1733 dans Epistle to Lord Cobham : 'Oh save my country! Heav' $n$ shall be your last'.

18 On sait en effet que le jardin fut un théâtre allégorique ou emblématique orchestré autour de temples et d'inscriptions ${ }^{33}$. La conception du jardin comme promenade ou déambulation se prêtait idéalement au progress, cette appréhension sérielle du sensible et du mental si typique de la Grande-Bretagne des Lumières. En laissant le promeneur "progresser " d'une étape à une autre, le jardin anglais invite à la réflexion, et le développement personnel de l'individu s'accompagne d'une méditation sur le " progrès » idéal de la nation. Individu et nation sont donc particulièrement imbriqués dans la réception de certains jardins anglais, comme si le jardin voulait être le carrefour d'un microcosme et d'un macrocosme. Aidé de ses amis poètes Pope et Lyttleton, ainsi que de Charles Bridgeman, John Vanbrugh, William Kent et Capability Brown, Lord Cobham exprima ses vues sur la nation en composant, ici avec des plantations, là avec des fabriques, des images satiriques et élogieuses. Le jardin contenait des éléments de panégyrique comme les sculptures de Georges $I^{\text {er }}$ (1723), du prince de Galles, futur Georges II (1724), et de la reine Caroline (1727), qui prouvent sa loyauté à l'égard de la dynastie des Hanovre nouvellement installée sur le trône national. Mais si Stowe fut 
"patriotique", c'est bien parce que le jardin fut moins emblématique de la gloire nationale que commentaire sur la nation. William Congreve rendit hommage à cette configuration «satirique» du jardin dans 'Of improving in the Present Time' (1728), et Lord Cobham le lui rendit bien en installant un monument à sa mémoire dessiné par Kent en 1736. Après 1733, date du vote de l'Excise Bill, Lord Cobham créa une faction de Boy Patriots et afficha son désaccord avec le premier des "Premiers ministres", Robert Walpole. La visite en 1737 du prince de Galles, Frederick, père de Georges III, cristallisa une opposition patriotique contre la gestion des affaires de Robert Walpole et la corruption des mœurs. Walpole fut perçu de plus en plus comme un suppôt du roi et de la dynastie des Hanovre, éloigné des intérêts de la nation. Le jardin connut là une instrumentalisation politique exemplaire ${ }^{34}$. Un temple de l'Amitié fut érigé en l'honneur du futur roi patriotique, 'Prince Frederick, Patriot King'; la statue du roi Georges II fut déplacée au début des années trente, tandis que le temple de la Vertu moderne exhiba un buste sans tête (certains y auraient reconnu là le Premier ministre).

D'un point de vue iconographique, le jardin repose sur une double mythologie, grécoromaine et gothique. La nation est célébrée par le prisme d'un culte indifférencié de la liberté, des saxons et des "Goths »: l'exèdre des déités saxonnes de Rysbrack, les bustes « anglais » de Gibbs (déplacés ensuite dans les niches du temple des 'British Worthies' de Kent), les "Champs-Elysées ", le temple de la Liberté. Plus tard dans le siècle, le jardin offrit des clins d'œil à Georges III : en 1763, à la fin de la guerre de Sept ans, le temple grec fut rebaptisé 'Temple of Concord and Victory' tandis que furent érigés un obélisque au général Wolfe (qui avait gagné le Canada à Québec) ainsi qu'un monument au capitaine Cook en 1778 (qui permit d'étendre l'empire jusqu'à l'Australie).

En conclusion, l'engagement personnel des figures royales et de l'aristocratie dans la promotion paysagère, avec l'aide notamment de Lord Burlington, de Lord Bute, et de Lord Cobham, révèle le désir d'exhiber un progrès de la nation. Il faut se souvenir de l'opposition entre True Patriots et False Patriots: certains jardins œuvraient pour le «progrès patriotique » qui mettrait le mieux en valeur la nation. Toutefois, ce mécénat patriotique ne doit pas occulter l'ironie de l'étiquette "jardin anglais» puisque les acteurs, les formes et les composantes de nombre de ces jardins étaient étrangers. La reine Caroline fut élevée à la cour de Sophie-Charlotte, électrice de Brandenbourg ; William Chambers eut un parcours international qui doit autant à la France, à l'Italie, à la Chine et à la Suède qu'à la Grande-Bretagne. Kew fut international dans ses références :

The message that Chambers was leading his visitors on a walk through world architecture is abundantly clear. Kew was the first of the World Fairs with their compression of world architecture! ${ }^{35}$

On ne peut pas non plus établir une équation entre le choix d'un style particulier et la nationalité éventuelle qu'il fut susceptible de dénoter. S'il ne fait pas de doute que la forme gothique fut reçue comme patriotique, il ne faut pas oublier que les Anglais l'avaient aussi découverte en Italie : comme l'a montré John Dixon Hunt, le gothique n'était pas nécessairement un crime de lèse-majesté vis-à-vis de l'Italie, qui a su honorer ce style. En outre, loin d'être uniformément classique ou gothique, Stowe exhiba, comme Kew Gardens, un large éventail de fabriques grecques, romaines, gothiques et chinoises, comme pour mieux souligner ${ }^{36}$ la part de liberté, de licence et de jeu que le propriétaire se réservait. Kent fit usage indifféremment de l'idiome gothique ou classique, conscient d'opérer dans les deux cas une translation: 
If natural settings and materials signalled gothic culture or Englishness, then surely Kent's sensitive siting of classical buildings in landscape groves or other natural contexts must be interpreted as one way in which he continued to mediate or to compromise between the two available styles: it was his way of making Rome talk 'good English', perhaps with some slight, affected Italian accent. ${ }^{37}$

Le terme «jardin anglais » masque donc la polysémie ou polyvalence de ses références et de ses sources architecturales. Cette polyphonie nous amène à rappeler les origines palimpsestueuses de ce prétendu « jardin anglais ».

\section{Le jardin anglais comme " bel infidèle » : traductions, translations et transpositions}

L'appellation « jardin anglais » met en valeur un savoir-faire, un talent, une esthétique là où il y eut enjeu, revendication et politique. Car le jardin s'est construit dans et par un discours sur la fierté nationale. C'est donc en termes de culture patriotique qu'il faut appréhender la dénomination « jardin anglais ». Le lieu le plus important où s'est élaboré le jardin anglais est peut-être moins le terrain lui-même que l'ekphrasis qui lui fut consacrée, et notamment celle de Horace Walpole, écrite et imprimée sur les presses de Strawberry Hill en 1771, avant d'être publiée séparément en 1780 : History of the Modern Taste in Gardening. En effet, sous couvert de retracer les origines d'une pratique à la mode dans le pays, Walpole fut un des premiers à jeter les bases d'une historiographie mensongère des jardins. Mais cette ekphrasis fit date car elle engendra un véritable palimpseste d'exégèses sur la nature du jardin et son authentique filiation. Ainsi Walpole fut secondé par son ami William Mason, qui composa un long poème, en quatre livres, entre 1772 et 1781 ; dans son édition du poème de 1783, William Burgh ajouta une glose avec des notes qui ne laissaient aucun doute sur la paternité du jardin anglais :

The Art is, therefore, our own, and consequently, the Poem, which, undertakes to

impart its principles, has a right to intitle itself the ENGLISH GARDEN ${ }^{\prime 38}$.

"Réduire ", comme on aurait dit au XVIII ${ }^{e}$ siècle, l'apport britannique dans la création paysagère, c'est identifier les sources, les dénominateurs communs, les radicaux. Or c'est précisément ce à quoi s'est refusé Walpole, donnant l'illusion d'une création ex nihilo. De nombreux historiens ${ }^{39}$ se sont interrogés sur ses motivations. Rappelons ici qu'il conféra à sa narration un sentiment artificiel de progrès comme si le jardin occidental avait traversé plusieurs phases de balbutiements avant d'atteindre la perfection emblématisée par William Kent sur le sol britannique. Ce qui a pris le pas sur la reconnaissance de dettes, c'est la naissance d'une nation, ou le mythe qu'une nation était née. Horace Walpole 'Patriot' se posa en "forgeron de la nation ", soucieux d'en établir de bonnes bases, mais soucieux aussi d'établir son identité en tant que fils (longtemps supposé bâtard) d'un père Premier ministre : 'Walpole's orientation was national'. ${ }^{40} \mathrm{Il}$ convient aussi de replacer sa revendication dans le contexte du débat sur l'origine, le génie et l'imitation : le jardin paraîtrait d'autant plus « original » qu'il serait « originel », sans précédents. On comprend pourquoi le jardin anglais fut littéralement une «invention». Enfin, on trouvera un dernier élément de réponse à nos interrogations en lisant la préface que Walpole écrivit pour ses Anecdotes of Painting in England. Il mit à nu le sentiment d'infériorité de sa nation et expliqua qu'il s'était senti forcé de modifier son « histoire de l'art anglais » en une « histoire de l'art en Angleterre» : 
This country, which does not always err in vaunting its own productions, has not a single volume to show on the works of its painters. In truth, it has very rarely given birth to a genius in that profession. Flanders and Holland have sent us the greatest men that we can boast. This very circumstance may with reason prejudice the reader against a work, the chief business of which must be to celebrate the arts of a country which has produced so few good artists. This objection is so striking, that instead of calling it The Lives of English Painters, I have simply given it the title of Anecdotes of Painting in England. As far as it answers that term, perhaps it will be found curious. ${ }^{41}$

Il nous parait légitime de concevoir que ce sentiment d'infériorité exhibé ici fut en retour compensé par un sentiment de supériorité qui fit du jardin anglais un unique mais singulier objet de gloire nationale. À force de "forger ", la narration fut faussée : sa History est une forgery, un faux. Ceci n'avait pas échappé à Uvedale Price en 1794:

It seems to me that there is something of patriotism in the praises Mr Walpole and Mr Mason have bestowed on English gardening; and that zeal for the honour of their country has made them, in the general view of this subject, overlook defects which they have themselves condemned..$^{42}$

Une telle perspective téléologique du jardin s'est faite au détriment d'une revendication qui aurait pu être plus originale : la Grande-Bretagne puisant aux sources de l'Europe. Mais le discours sur le jardin anglais a servi de masque pour dissimuler les emprunts au lieu de les revendiquer. Si l'on reprend les prétendues caractéristiques du jardin anglais, on verra qu'elles ont depuis été attribuées à d'autres nations ${ }^{43}$. La tradition paysagère anglaise aurait bien pu s'appeler traduction(s). Tout d'abord, le ha-ha: 'I call a sunk fence the leading step [...] he leaped the fence, and saw that all nature was a garden'. Proclamé par Walpole comme étant le coup de génie de Kent, il doit être rendu à Charles Bridgeman qui en fit usage le premier en Angleterre, mais aussi et surtout à la France, puisqu'on sait bien maintenant que le saut-de-loup fut une pratique française, inspirée des fortifications militaires.

En second lieu, Walpole argue de l'ouverture vers la campagne alentour. Or c'est une des traditions italiennes du jardin, comme on peut le constater par exemple à Florence dans les jardins Boboli où l'étagement en terrasses jette progressivement l'œil de plus en plus loin. En troisième lieu, les qualités picturales et théâtrales que Walpole attribua à Kent devraient être «traduites» de manière plus neutre en termes de l'incidence sur le sol anglais de la tradition scénique des jardins italiens:

Kent's painterly and theatrical handling of space was susceptible to interpretation in progressive ways that satisfied a later taste. Yet it was equally indebted, we can be sure, to Kent's experience of older Italian gardens. ${ }^{44}$.

En quatrième lieu, il a été dit que le jardin anglais se distingue par l'asymétrie et un respect de la nature, mais ce choix de ne plus tirer au cordeau a été motivé par des réminiscences de pratiques chinoises. Ce qu'on nomme l'irrégularité « anglaise » devrait être retraduite dans son idiome pseudo-originel qui est le sharawadgi. En effet, à la fin du XVII siècle, William Temple fut un des premiers - dès 1685 dans Upon the Gardens of Epicurus - à préconiser un renouveau en s'appuyant sur ce mot oriental (que depuis on rapproche du sorowaji japonais ${ }^{45}$ ) : il n'avait toutefois pas éprouvé le besoin d'angliciser le terme. Par la suite, ses idées furent véhiculées par Addison dans ses essais dans The Spectator et le sharawadgi fut naturalisé en Angleterre.

En dernier lieu, on peut soutenir que la manière dont Thomas Whately dans ses Observations on Modern Gardening (1770), reprises en partie par Walpole, compartimenta 
ses descriptions de jardin selon une combinaison de minéraux, de végétaux et d'eau, fut inspirée du discours oriental sur le jardin ; celui-ci avait pu se répandre oralement dans les années 1740 par le général George Anson, le créateur de Shugborough ou par William Chambers, avec son essai 'On the Art of Laying out Gardens among the Chinese' (1757) ${ }^{46}$. Le vocabulaire que Walpole s'entêta à présenter comme indigène était donc une traduction de pratiques ancestrales étrangères que les Anglais avaient translatées sur leurs sols de manière - incontestablement - originale ; mais l'emprunt masqué lui, peut aujourd'hui en ces temps historicistes paraître contestable. Car au départ c'est bien au nom des pratiques en Orient que se fit l'émancipation britannique hors des textes et des contextes français et hollandais. Le jardin est donc "bel infidèle ", telle une traduction occidentale des Mille et une Nuits qui s'immisce tout en transposant le «costume». Le discours négatif et négateur de Walpole viendrait-il confirmer la thèse d'Edward Saïd selon laquelle l'empire britannique, loin de se ressourcer aux puits de l'orient, fit preuve d'annexion dans sa volonté impérialiste et nationaliste d'assimilation?

\section{Les chinoiseries franco-anglaises : la querelle aux enjeux nationalistes distincts}

31 Le jardin anglais est une expression parmi d'autres choisies au milieu du XVIII ${ }^{e}$ siècle en Angleterre par les «nationalistes » pour fixer des notions fluctuantes. Comme l'atteste le concept du beau, l'esthétique a parfois du mal à définir ses catégories. Ce qu'on nomme aujourd'hui en Europe 'English garden' ou «jardin à l'anglaise » aurait pu être connu sous le terme plus neutre de jardin paysager. On en prendra pour preuve la confusion terminologique entre 1720 et 1780 pour désigner la nouvelle manière de composer les jardins en Grande-Bretagne ; appelés 'modern', ils furent aussi affublés, selon que l'on était d'un côté ou de l'autre de la Manche, des qualificatifs 'English', 'Landscape Garden', « anglochinois », " cochin-chinois ", 'Picturesque' et " pittoresque ». Walpole eût été plus honnête s'il avait conservé la terminologie qu'il employa en note en 1771 dans ses Anecdotes of Painting:

Note: I have not been able to please myself with a single term that will express ground laid on principles of natural picturesque beauty, in contradistinction to symmetrical gardens - but I am very clear that the designer of modern improvements in Landscape-Gardens (as I will call them for want of a happier appelation) ought by no means to be confounded with the domestic called a Gardiner. ${ }^{47}$

Le jardin anglo-chinois est devenu " jardin anglais » par un nationalisme abusif ${ }^{48}$. Pour le voyageur français souvent cité, Pierre-Jean Grosley, le "goût actuel des Anglais pour l'arrangement de leurs parcs et de leurs jardins est dans le goût chinois, c'est à dire le goût qui comme tous ceux de cette nation est établi chez elle de toute antiquité $"^{49}$. Dès lors que William Chambers imprima ses pensées sur la distribution des jardins en Chine, en 1757, puis en 1772-73, les partisans du jardin anglais autour de la figure de Walpole entrèrent en guerre pour fixer à jamais l'origine britannique du jardin, et éviter que le point de vue plus "diffus» de Chambers ne "noie» la créativité nationale dans une masse internationale indistincte. Thomas Gray s'était inquiété :

The only taste we can call our own, the only proof of our original talent in matters of pleasure; I mean, our skill in gardening, \& laying out grounds. that the Chinese have this beautiful Art in high perfection, seems very probable from the Jesuits's letters, \& more from Chambers's little discourse published some few years ago, but 
it is very certain, we copied nothing from them nor had anything but nature for our model. It is not forty years, since the Art was born among us; and it is sure, that there was nothing in Europe like it. \& as sure, we then had no information on this head from China at all. ${ }^{50}$ revendiquèrent de nouvelles tendances. Ce qui importe ici, c'est de comprendre pourquoi le jardin en Grande-Bretagne ne s'épanouit pas au nom d'un goût pour l'Orient, qu'il fût authentique ou faussé. Chambers fit cette référence, ainsi que William Beckford, par exemple, à qui Chambers avait offert sa Dissertation en 1773. Que révèle le fait que l'influence orientale ait été occultée et ait causé une telle division nationale ? Pourquoi Walpole nia-t-il l'inspiration chinoise après avoir lui-même avoué être sous le charme de la Chine ? La correspondance de Walpole avec Mason révèle qu'il le fit pour défendre la nation ('To continue an Englishman'). En effet, le culte d'une certaine virilité et d'une 
simplicité, ainsi que l'obsession de la pureté que l'Angleterre lègua à la France au début du XIX ${ }^{e}$ siècle, annihilèrent la légitimité du goût chinois qui s'apparentait trop au rococo français. Walpole et ses amis, contrairement à un Beckford, n'auraient-ils pas su distinguer les chinoiseries du goût oriental ? Un autre élément d'ordre idéologique vient expliquer l'étiquette « jardin anglais ». Horace Walpole, aidé de Mason, comptait défendre une idée de l'anglicité et de la nation qui était plus étroite et insulaire que celle de Georges III et du francophile Chambers. Entendons une dernière fois Walpole, qui livre dans une de ses notes manuscrites le cœur de son idéologie :

At least it will show what a Paradise was England while She retained her Constitution for perhaps it is no paradox to say,$^{54}$ that the reason why Taste in Gardening was never discovered before the beginning of the present Century, is, that It was the result of all the happy combinations of an Empire of Freemen, an Empire formed by Trade, not by a military \& conquering Spirit, maintained by the valour of independent Property, enjoying long tranquillity after virtuous struggles, \& employing its opulence \& good Sense on the refinements of rational Pleasure. ${ }^{55}$

Deux camps semblent donc s'être opposés pour s'approprier le « véritable patriotisme»: les "nationaux chauvins" et les "nationaux cosmopolites", pour ainsi dire. La dimension internationale d'un Chambers était aux antipodes des choix culturels de nombreux artistes comme Hogarth, Brown ou Repton, au grand dam d'autres, dilettanti ou poètes, comme Kent, Richard Payne Knight, Beckford et Blake, qui s'accommodaient parfaitement de l'acte de translation et de traduction.

\section{Conclusion}

41 Cet article a souhaité mettre en relief les inadéquations entre jardin et nation dissimulées par l'étiquette « jardin anglais ». Sans pour autant nier la modernité du mythe horticole de la Grande-Bretagne, nous avons souligné les mécanismes de mise en place de mythes, tout en relevant les enjeux sociaux et politiques. Quand les Français traduisirent 'English garden' par " anglo-chinois ", les Anglais soutinrent qu'ils « chinoisaient »; pourtant ceux qui chinoisèrent véritablement sont bien les Anglais puisque la manière dite naturelle qu'ils revendiquaient était, sinon empruntée à l'art chinois ou inspirée de lui, du moins affiliée à cet art. Nombre de plans de jardins de la fin du XVIII ${ }^{e}$ siècle portent le nom de jardin anglais ou " anglois » : ce terme avait donc un sens pour les contemporains, même si ceux-ci n'auraient pas nécessairement pu en donner une définition unique et claire. On ne saurait néanmoins fixer ni réduire en termes lexicaux une réalité qui en contenait plusieurs. L'objet de cet article n'était ni d'apporter une définition supplémentaire au « jardin anglais », ni de nier la pertinence de cette étiquette ; il a rappellé l'usage qu'il en a été fait des deux côtés de la Manche, et a tenté de démêler l'écheveau des enjeux patriotiques et proto-nationalistes qui s'y rattachent. 


\section{NOTES}

1. J.D. HUNT, Gardens and Groves : The Italian Renaissance Garden in the English Imagination, 1600-1750, London: J.M.Dent \& Sons, 1986, p. 180 et M. BARIDON, Le Jardin paysager anglais au siècle des Lumières, Dijon: EUD, 2000.

2. Horace WALPOLE, The History of the Modern Taste in Gardening (imprimé sur les presses privées de Strawberry Hill en 1771 mais publié à Londres en 1780), ed. J.D. HUNT, New York: Ursus Press, 1995, pp. 56-57.

3. Voir G.E. FUSSELL, Landscape Painting and the Agricultural Revolution, London: The Pindar Press, 1984, Nigel EVERETT, The Tory View of Landscape, London: Yale University Press, 1994 (notamment 'The Whig Idea of Landscape and its Critics', pp. 38-91 et Polite Landscapes Gardens and Society in Eighteenth-Century England, Baltimore: The Johns Hopkins University Press, 1995).

4. Jacques CARRÉ, «Le concept d'improvement et les élites foncières anglaises à l'époque de la révolution agricole ", Cercles, Actes du colloque de l'université de Rouen (10 décembre 1994) sur les élites foncières en Angleterre du milieu du XVIII ${ }^{\text {e }}$ siècle à 1846, Rouen, Université de Rouen, 1995, pp. 39-52 ; Suzy HALIMI, «La maison de campagne, apanage des élites foncières en Angleterre au XVIII ${ }^{e}$ siècle ", XVII-XVIII : Bulletin de la société anglo-américaine d'études sur les $\mathrm{XVII}^{\mathrm{e}}$ et XVIII ${ }^{\mathrm{e}}$ siècles, $\mathrm{n}^{\circ} 39$ (1994), pp. 7-20. On associe un peu trop récemment le goût de la propriété à l'ère thatchérienne, alors que c'est elle qui s'inspira de l'aristocratie foncière des XVII ${ }^{\mathrm{e}}$ et XVIII ${ }^{\mathrm{e}}$ siècles, comme l'atteste la caricature dérivée de Mr and Mrs Andrews de Gainsborough, où le couple offre un os à un chien portant la tête de M. Thatcher (Peter BROOKES d'après Gainsborough, 'Mr and Mrs Andrews with Margaret', The Spectator, 22 October 1988 ; utilisé par Stephen DANIELS, Fields of Vision : Landscape Imagery and National Identity in England and the United States, Princeton: University Press, 1993, p. 105)

5. John BARRELL, The Birth of Pandora and the Division of Knowledge, Basingstoke: Macmillan, 1992 et William John MITCHELL, Landscape and Power, Chicago: University Press, 1994.

6. Augustin BERQUE a proposé plusieurs degrés de maturité d'une civilisation paysagère dans Les Raisons du Paysage : De la Chine antique aux environnements de synthèse, Paris : Hazan, 1995, pp. 34-35: «1) des représentations linguistiques (un mot ou des mots pour dire 'paysage') ; 2) des représentations littéraires, orales ou écrites; 3) des représentations picturales; 4) des représentations jardinières"

7. S. DANIELS, Fields of Vision, p. 82.

8. L. COLLEY, Britons : Forging the Nation, 1707-1837, London: Pimlico, 2003, p. ix.; ou encore, 'in the eighteenth century, as after, men and women shuffled identities like cards, feeling exclusively English or Scottish or Welsh in some circumstances perhaps, but choosing to define themselves as British in others' (p. x).

9. S. DANIELS, Fields of Vision, p. 6.

10. 'It could be argued that for Scotland, at least, the landscape garden was an aberration, forced upon the country through its close association with England after the Union of the Parliaments in 1707, and that temperamentally, historically, and in practical terms the formal garden of the seventeenth-century, seen from Aberdourto Balcaskie, was the ideal national form.' (A.A. TAIT, The Landscape Garden in Scotland, 1735-1835, Edinburgh: University Press, 1980, p. 239). Voir également Finola O'KANE, Landscape Design in Eighteenth-Century Ireland : Mixing Foreign Trees with the Natives, Cork: University Press, 2004. 
11. Todd LONGSTAFFE-GOWAN, The Gardens and Parks at Hampton Court Palace, London: Frances Lincoln, 2005, p. 13.

12. David JACQUES \& A. Jan van der HORST, The Gardens of William and Mary, London: Helm, 1988, 'Dutch Gardens in Britain', p. 97.

13. 'A lively interest in French engravings of garden architecture and ornament [...which] served to encourage many to adopt the layout, decoration and iconography of French gardens.' (Todd LONGSTAFFE-GOWAN, The Gardens, p. 81)

14. D. JACQUES \& A. Jan van der HORST, The Gardens, p. 99.

15. Stephen SWITZER, Ichnographia Rustica : or, the Nobleman's, Gentleman's, and Gardener's Recreation, London, 1718, p. 57.

16. Voir les travaux de Michel Baridon sur l'incidence de Locke et de Hume sur la réception du jardin anglais par le promeneur; Le Jardin anglais (Dijon : Presses Universitaires de Dijon, 2000).

17. The Gentleman's Magazine, VII (May 1737), pp. 917-918.

18. John BARRELL, The Dark Side of the Landscape : The Rural Poor in English Painting, 1730-1840. Cambridge: University Press, 1980.

19. Cosgrove note qu'à Blenheim les classes laborieuses furent dotées d'un village modèle, ancêtre de l'utopie de la cité-jardin, mais à l'écart du domaine : 'Screened by well-placed trees is the model village built for the labourers who invisibly maintained this country garden (...) But the people who produced these benefits, the 60 persons, for example, permanently employed to cater for Blenheim's 2,500 acres, are entirely excluded from its "landscape" '. (Denis COSGROVE, Social Formation and Symbolic Landscape (1984), Wisconsin: University of Wisconsin Press, 1998, pp. 214-215).

20. Denis COSGROVE, Social Formation, p. 205.

21. 'In 1816 Repton recalled a time when his art "seemed likely to extend its influence till all England would become one landscape garden"; "but eager pursuit of gain has, of late, extended from the new proprietor, whose habits have been connected with trade, to the ancient hereditary gentleman" '. (Stephen DANIELS, 'Humphry Repton and the Improvement of the Estate', in Fields of Vision, pp. 80-111.)

22. William COBBETT, 'Address to the People of the United States of America', Cobbett's Weekly Political Register, 18 May 1816 ; également cité in DANIELS, Fields of Vision, p. 99.

23. Les guillemets sont là pour rappeler l'anachronisme qui consiste à utiliser le terme « nationaliste » pour le XVIII ${ }^{\mathrm{e}}$ siècle, mais étant donné l'exacerbation grandissante des egos des nations à la fin du XVIII ${ }^{e}$ siècle, nous employons ici indifféremment «nationaliste » et " patriotique ». La recherche récente s'illustre par Howard WEINBROT, Britannia's Issue : The Rise of English Literature from Dryden to Ossian, Cambridge: University Press, 1993 ; Colin KIDD, British Identities before Nationalism : Ethnicity and Nationhood in the Atlantic World, 1600-1800, Cambridge: University Press 1999 ; et Dustin GRIFFIN, Patriotism and Poetry in EighteenthCentury Britain, Cambridge: University Press, 2002, pp. 7-33.

24. Holger HOOCK, The King's Artists : The Royal Academy of the Arts and the Politics of British Culture, 1760-1840, Oxford: Clarendon Press, 2003.

25. En 1976, Judith COLTON écrivait 'But Queen Caroline's effort to promote the arts was to remain unique in England. The Hanoverian Kings had little use for statuary and even less for gardens' (Judith COLTON, 'Kent's Hermitage for Queen Caroline at Richmond', Architectura, IV (1976) pp. 181-191) ; de nombreuses études apportent depuis un démenti.

26. Lettre de Thomas Robinson au comte de Carlisle, 1734, Historical Manuscript Commission Reports, Castle Howard MSS, VI (1897), pp. 143-4 (quoted by J.D. Hunt, William Kent - Landscape Garden Designer : An Assessment and Catalogue of his Designs, London: A. Zwemmer, 1987, p.46). 27. Lettre du pépiniériste Peter Collinson à John Bartram en Amérique du Nord, datée du 24 avril 1751 et lettre de John Mitchell, datée du 30 avril 1751, citées par John HARRIS \& Michael SNODIN, Sir William Chambers Architect to George III, Yale University Press, 1996, p. 57. 
28. Voir Judith COLTON, 'Kent's Hermitage for Queen Caroline at Richmond', Architectura, IV (1976) 181-191 et 'Merlin's Cave and Queen Caroline: Garden Art as Political Propaganda', Eighteenth-Century Studies, 10 (1976-77), pp. 1-20, ainsi que J.D. HUNT, William Kent, pp. 61-65.

29. The Gentleman's Magazine, II (August 1732), pp. 917-918.

30. The Gentleman's Magazine, II (August 1732), p. 923.

31. Cité dans The Gentleman's Magazine, III (August 1733), pp. 421-422.

32. Richard DRAYTON, Nature's Government: Science, Imperial Britain, and the 'Improvement' of the World,Yale University Press, 2000, p. 68.

33. Voir John Martin ROBINSON, Temples of Delight (Andover: Pitkin Pictorials Ltd, 1990), Suzy HALIMI, «Lecture d'un jardin anglais : Stowe», XVII-XVIII-Bulletin de la société angloaméricaine d'études sur les XVII ${ }^{\mathrm{e}}$ et XVIII ${ }^{\mathrm{e}}$ siècles 51 (nov. 2000), pp. 151-166 et Stowe Landscape Gardens : A Comprehensive Guide (London: The National Trust, 2005).

34. Tom Williamson choisit de minimiser la lecture politique du jardin anglais - aussi bien que philosophique - en rappelant que tous les propriétaires n'avaient pas l'agenda de Lord Cobham et que les dessins paysagers résultèrent plus souvent qu'on ne le croit d'humeurs, de goûts horticoles et de décisions personnelles qui ne nous seront parfois jamais connus ; voir Tom WILLIAMSON, Polite Landscapes, p. 65 : 'Yet we must be careful not to exaggerate the importance of politics, and especially of one political clique, in the development of the landscape garden in the 1730s and '40s. Symbolic gardens were no monopoly of the opposition. [...] Gardens could now be used as media to vent all sorts of highly personalized views of the world, political or otherwise.'

35. J. HARRIS \& M. SNODIN, William Chambers, p. 67.

36. Voir notre "'Of Greeks and Goths in the English Garden' : sources 'classiques' et sources 'modernes' dans l'Angleterre de la fin du XVIII' siècle », XVII-XVIII, n60 (2005), pp. 217-242.

37. J.D. HUNT, William Kent, p. 65.

38. William BURGH, in William MASON, The English Garden : A Poem In Four Books (1772-1781). To Which is added a commentary and notes by William Burgh, London (1783), p. 208.

39. Dora WIEBENSON, The Picturesque Garden ; John Dixon HUNT, Garden and Grove et William Kent : Landscape Garden Designer ; Stephen BENDING, 'Horace Walpole and Eighteenth-Century Garden History', Journal of the Warburg and Courtauld Institutes, 57 (1994), pp. 209-222.

40. Dora WIEBENSON, The Picturesque Garden in France, Princeton: UP, 1978, p. 61.

41. Horace WALPOLE, Anecdotes of Painting, London: J.Dodsley, 1786, $3^{\text {rd }}$ edition, vol 1, ii.

42. Uvedale PRICE, Essay on the Picturesque as compared with the Sublime and the Beautiful, London, 1794-98, vol. I, p. 275.

43. Voir notre Jardin et paysage en Grande-Bretagne au XVIII ${ }^{\mathrm{e}}$ siècle (avec Marie-Madeleine Martinet), Paris : CNED, 2001.

44. J.D. HUNT, William Kent : Landscape Garden Designer, p. 46.

45. Ciaran MURRAY, 'Sharawadgi resolved', Garden History, vol. XXXVI, n²2 (1998), pp. 208-213.

46. Notons que le traducteur français des Observations de Whately inséra une traduction du texte de Chambers dans son édition, L'Art de former les jardins modernes ou l'art des jardins anglais (1771).

47. WALPOLE, Anecdotes of Painting, vol. 4, chap. III, 'Painters in the Reign of George II', p. 106.

48. La bibliographie sur la controverse du jardin anglo-chinois est la suivante: Arthur O. LOVEJOY , 'The Chinese Origin of a Romanticism', Journal of English and Germanic Philology (janvier 1933) ; Dora WIEBENSON, The Picturesque Garden ; Patrick CONNER, 'China and the Landscape Garden : Reports, Engravings and Misconceptions', Art History, vol. 2, n 4, décembre 1979, pp. 429-440 ; David JACQUES, 'On the supposed Chineseness of the English Landscape Garden', Garden History , vol. LVIII, n² (1990), pp.181-191; Michel BARIDON, Le Gothique des Lumières, Brionne : G. Monfort, 1991; Monique MOSSER (introduction), Aux jardins de Cathay: L'imaginaire anglochinois en Occident, Paris : Éditions de l'Imprimeur, 2004, pp. 11-127. 
49. GROSLEY, Londres, 1770, vol III, pp. 105-29.

50. Thomas Gray à William Taylor How, in T. GRAY, Correspondence of T. Gray, eds. Paget TOYNBEE \& Leonard WHIBLEY, Oxford, 1971, vol. 11, p. 813, '10 Sept. 1763'.

51. The Correspondence of Horace Walpole, Earl of Orford and the Reverend William Mason, now published from the original MSS, ed. John MITFORD, London: Richard Bentley, 1851, 'May $9^{\text {th }}$, 1772', pp. 22-23.

52. Extrait d'une note sur Brown in Satirical Poems Published Anonymously by William Mason with Notes by Horace Walpole, now first printed from his MS, ed. Paget TOYNBEE, Oxford: Clarendon Press, 1926, p. 41.

53. David PORTER, 'From Chinese to Gothic : Walpole and the Gothic Repudiation of Chinoiserie', Eighteenth-Century Life (Feb. 1999), p. 47.

54. En italiques ensuite dans le MS.

55. Satirical Poems [...] with Notes by Horace Walpole, now first printed from his MS, op. cit., p. 44.

\section{RÉSUMÉS}

Convoquer de concert les notions de jardin et de nation, c'est inscrire la création paysagère dans son histoire, c'est-à-dire dans son contexte social, culturel et politique, mais c'est aussi introduire une réflexion linguistique sur les modalités d'appropriation nationale, voire «nationaliste » du jardin. S'il est indéniable que le jardin contribue à la modernité de la Grande-Bretagne, que la nation s'est construite une image grâce au jardin, il reste qu'en cette ère critique du soupçon on ne saurait être prisonnier des mots; le jardin recouvre bien plus qu'une créativité proprement indigène : c'est aussi un discours d'auto-promotion nationale et une traduction-translation de modes étranger(e)s. Cet article étudie le le jardin comme icône nationale, soulève le problème de l'anglicité et du jardin "anglais » comme une image partielle de la nation et du jardin comme représentation «nationaliste » ('A Patriotick Progress?') ; il propose ensuite de concevoir le jardin anglais comme «bel infidèle» en soulignant les traductions, translations et transpositions à l'œuvre dans le discours ekphrastique mais aussi les chinoiseries franco-britanniques, avant de conclure sur les enjeux nationalistes distincts au sein même de l'Angleterre des Lumières.

Bringing the idea of nation to bear on that of garden is a way of placing landscaping in its history, namely its social, cultural and political context, but it is also a way of understanding how national, or even 'nationalist', appropriation functions. While it is undeniable that gardens contributed to the rise of modernity in Great-Britain, and that the new nation built itself an image thanks to the garden, one should not be blind to words: gardens are much more than just native skill, and correspond to a discourse of national self-promotion, as well as a translation of foreign mores. This article explores the garden as national icon, raising the problem of the 'Englishness' of the English garden by showing how the so-called English garden was at times a partial image of the nation and at other times an overtly nationalistic representation ('A Patriotick Progress?'). It then offers to see the English garden as one of those 'beautiful infidels' typical of Enlightenment translations, pointing out the way ekphrastic commentaries made use of foreign terms and traditions. Lastly, the article highlights the bickering that took place between France and Britain over the terminology apt to describe the jardin anglois, the rôle of 
China in shaping both discourse and practice of gardening in Britain, and the import of distinct nationalist ideologies that prevailed in England itself at the time.

INDEX

Mots-clés : jardin anglais, XVIIIe siècle, Chine

Keywords : English garden, 18th century, China

\section{AUTEUR}

LAURENT CHÂTEL

Université Paris IV-Sorbonne 